\title{
THE ASSOCIATION OF SEDATIVES AND ANTICONVULSANTS WITH AEEG RECOVERY IN ASPHYXIATED TERM INFANTS RECEIVING HYPOTHERMIA TREATMENT
}

\author{
A.J. Csekő ${ }^{1}$, P. Lakatos ${ }^{1}$, J. Kárdási ${ }^{1}$, L. Pusztai ${ }^{1}$, B. Hallberg ${ }^{2}$, M. Szabó ${ }^{1}$ \\ ${ }^{1} 1$ st Department of Pediatrics, Semmelweis University, Budapest, Hungary, ${ }^{2}$ Karolinska Institutet and \\ University Hospital, Stockholm, Sweden
}

Background: Both hypothermia and CNS drugs may alter the prognostic accuracy of aEEG in HIE. We aimed to assess the predictive value of aEEG in hypothermia treated HIE infants. Furthermore we intended to investigate the association of cumulative doses of CNS drugs with aEEG recovery.

Patients and methods: 63 term HIE infants treated with moderate systemic hypothermia for 72 hours were continuously monitored by single-channel aEEG. Records were evaluated according to the classification suggested by Hellström-Westas (2006., Seminars in Fetal \& Neonatal Medicine). Doses of administered Morphine, Phenobarbitone and Midazolam were recorded. Poor outcome was defined as death or severe neurodevelopmental delay at 18-22 months (Bayley Scales of Infant Development II), good outcome as absence of these criteria. Mann-Whitney U test was used for statistical analysis.

Results: Poor outcome $n=25$, good outcome $n=38$. PPVs of an abnormal background pattern to predict poor outcome were 0.5 at $6 \mathrm{hs} ; 0.7$ at $24 \mathrm{hs} ; 0.88$ at $48 \mathrm{hs}$ and 0.95 at $60 \mathrm{hs}$. Cumulative doses of the investigated drugs did not differ between infants with onset of normal background pattern before and after 24 hours.

Conclusions: aEEG provides the best prediction of outcome from the 48th hour during hypothermia in HIE infants. Commonly used CNS drugs in HIE infants do not seem to considerably delay aEEG recovery.

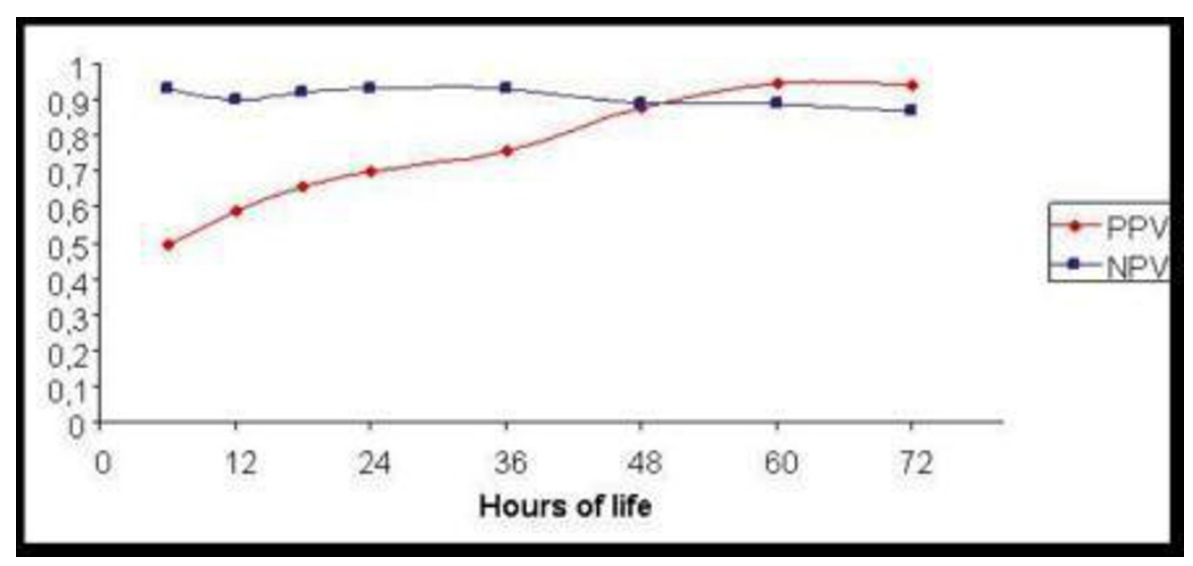

[Predictive values of aEEG in hypothermia] 